\title{
Analysis of the Prompt Fluorescence Induction by Means of Computer Simulation of the Primary Photosynthetic Reactions
}

\author{
Christian Holzapfel \\ Institut für Chemie II, Kernforschungsanlage Jülich \\ Z. Naturforsch. 33 c, 402-407 (1978) ; received April 17, 1978 \\ Primary Photosynthetic Electron Transport, Computer Simulation, Fluorescence Induction, \\ ATP-Formation
}

The induction phenomenon of prompt fluorescence of the photosynthetic system in green plants reflects the adaptation of the electron transport system from a dark adapted state to a steady state under light condition. The appearance of the P-peak in the prompt fluorescence induction curve is explained by the decay of the electrochemical gradient across the thylakoid membrane in the dark. The decay of the electrochemical gradient in the dark is calculated using measured fluorescence induction curves with different times of dark adaptation. The influence of photophosphorylation inhibitors is discussed and the threshold effect of ATP-formation depending on the electrochemical gradient is explained by the coupling of the ATP-formation with the electron transport in the thylakoid membrane.

\section{Introduction}

The intensity of prompt fluorescence from photosystem II in the photosynthetic system of green plants reflects the redox state of the quencher Q, the primary electronacceptor of photosystem II [1 - 4]. The redox state of the quencher Q depends on the state of all the other pools in the electron transport chain from the first electron donor of photosystem II - the water splitting system $Z$ - to the terminal electron acceptor of photosystem I - probably ferredoxin - which reduces $\mathrm{NADP}^{+}$. This process is described by the Z-scheme by Bendall and Hill [5]. The electron transfer causes an electrochemical potential difference across the thylakoid membrane providing energy for the synthesis of ATP from ADP and inorganic phosphate [6-8]. ATP and reduced NADP drive the dark reaction, i.e., the reduction of $\mathrm{CO}_{2}$ to $\left(\mathrm{CH}_{2} \mathrm{O}\right)_{6}$ in the Calvin-Benson cycle. The induction phenomena of prompt fluorescence of green plants subjected to a sudden change from dark to light reflect the change of the redox state of the quencher $Q$ during adaptation of the whole system from the dark adapted state to the steady state in the light. This can be described by a computer model in terms of coupled redox pools of the intermediates of the electron transport chain $[9,10]$. The change of reduced quencher $\mathrm{Q}^{-}$shows the characteristics of the fluorescence induction curve as measured e.g. in green algae - the Kautsky effect.

Requests for reprints should be sent to Dr. Chr. Holzapfel, Institut für Chemie II, Kernforschungsanlage Jülich GmbH., Postfach 1913, D-5170 Jülich.
The peak $\mathrm{P}$ is caused by a pile up of electrons in the chain due to the initial inability to transport electrons, probably because of a lack of ATP necessary to reoxidize $\mathrm{NADPH}_{2}$. As ATP is synthesized by the increasing electrochemical potential difference, $\mathrm{NADPH}_{2}$ and also the other pools are reoxidized due to the increasing electron flow through the chain. Therefore, this high energy state should lead to the decrease of fluorescence intensity from peak $\mathrm{P}$ to steady state $\mathrm{S}$ (curve 0 in Fig. 5), probably by reoxidating $\mathrm{NADPH}_{2}$ via ATP formation. Thus it may be possible to use the fluorescence induction curve, and especially the peak $\mathrm{P}$, as an indicator of the high energy state in the photosynthetic system. In this paper the decay of the electrochemical potential in the dark is calculated from the measured height of the P-peak in the induction curve after different times of dark adaptation.

\section{Methods and Materials}

The photosynthetic sample used was a suspension of the green algae Chlorella vulgaris grown in aerated liquid culture illuminated by white fluores. cence tubes. The culture medium had the following composition per liter (slightly modified from [24]) : $10^{-2} \mathrm{~mol} \mathrm{KNO}_{3}, 4.52 \times 10^{-3} \mathrm{~mol} \mathrm{NaH}_{2} \mathrm{PO}_{4}, 5.3 \times$ $10^{-4} \mathrm{~mol} \mathrm{Na}_{2} \mathrm{HPO}_{4}, 1.6 \times 10^{-5} \mathrm{~mol} \mathrm{H}_{3} \mathrm{BO}_{3}, 2.5 \times$ $10^{-5} \mathrm{~mol}$ EDTA (Titriplex III), $10^{-3} \mathrm{~mol} \mathrm{Mg}^{2+}$, $10^{-4} \mathrm{~mol} \mathrm{Ca}{ }^{2+}, 2.5 \times 10^{-5} \mathrm{Fe}^{2+}, 10^{-6} \mathrm{~mol} \mathrm{Zn}^{2+}$, $7 \times 10^{-7} \mathrm{~mol} \mathrm{Mn}^{2+}, 2.4 \times 10^{-8} \mathrm{~mol} \mathrm{Cu}{ }^{2+}, 8.4 \times$ $10^{-9} \mathrm{~mol} \mathrm{Co}{ }^{2+}, 10^{-3} \mathrm{~mol} \mathrm{SO}_{4}{ }^{2-}$, and $10^{-4} \mathrm{~mol} \mathrm{Cl}{ }^{-}$. $\mathrm{pH}$ was about 6 . The glass tubes with the culture were flushed with air from the bottom and il- 
luminated by 5 white light fluorescence tubes $(40 \mathrm{~W})$ in a distance of about $15 \mathrm{~cm}$ with a light-dark period of 14:10 hours. The prompt fluorescence is measured in the usual way exciting the sample with the light of a $100 \mathrm{~W}$ halogen lamp through a blue filter (BG18 or SFK6, Schott/Mainz) and recording the signal through an interference filter at $650 \mathrm{~nm}$ by a photomultiplier (EMI 9683) and a storage oscilloscope. The experimental results were compared with the computer model stored on the IBM/378-168 at KFA Jülich [10].

\section{Description of the Computer Simulation of the Photophosphorylation}

The Z-scheme of the electrontransport in the thylakoid membrane can be described in terms of coupled redox pools of the intermediates. Electrons are pumped by the two photosystems PSII and PSI (Fig. 1) from the first water splitting electron donor $\mathrm{Z}$ of PSII to the terminal electron acceptor of PSI, Ferredoxin (FD) and $\mathrm{NADP}^{+}$, from where the dark reactions start. According to the chemi-osmotic theory ATP is synthesized from ADP using the energy stored in the electrochemical potential difference across the thylakoid membrane. This electrochemical potential difference across the membrane is built up by $\mathrm{H}^{+}$-transport across the membrane coupled to electron transport $f_{\mathrm{e}}$ in the membrane $[11,12]$. The water splitting process results in a release of one $\mathrm{H}^{+}$-ion at the inside of the membrane for each electron transported to $Z$. The reduction of $\mathrm{PQ}$ at the outside of the membrane is as-

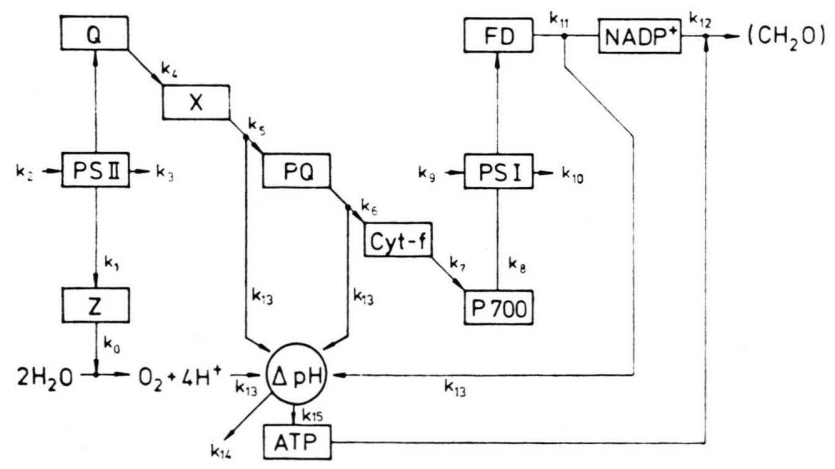

Fig. 1. Z-scheme of the photosynthetic electron transport chain from water to $\mathrm{NADP}^{+}$represented as a compartment model of electron pools. PSII and PSI, photosystem II and I; Z, water splitting system of PSII (primary donor); Q, quencher of fluorescence (primary acceptor of PSII); X, PQ (plastoquinone), and Cyt-f, intermediate pools; P700, reactioncenter chlorophyll $\mathrm{a}_{700}$ of PSI; FD, ferredoxin. sociated with an uptake of one $\mathrm{H}^{+}$-ion from the stroma for each electron transported from $\mathrm{X}$ to $\mathrm{PQ}$. The reoxidation of $\mathrm{PQH}_{2}$ at the inside of the membrane releases one $\mathrm{H}^{+}$-ion into the interior of the thylakoids for each electron transported from $\mathrm{PQ}$ to Cyt-f. Finally, the reduction of $\mathrm{NADP}^{+}$in the stroma results in an uptake of one $\mathrm{H}^{+}$-ion from the stroma for each electron transferred from FD to $\mathrm{NADP}^{+}$. Altogether this means that two $\mathrm{H}^{+}$-ions are taken up from the stroma outside and two $\mathrm{H}^{+}$-ions are released to the inside of the thylakoids, i.e. a net transport of two $\mathrm{H}^{+}$-ions through the membrane for each electron transferred through the whole transport chain from $\mathrm{Z}$ to $\mathrm{NADP}^{+}$. As a consequence $\mathrm{pH}$ decreases inside the thylakoids and increases outside the thylakoids. The simplest way to describe this is to assume that the $\mathrm{pH}$ difference is proportional to the electron flow rate. The $\mathrm{pH}$-difference also leads to a backflow of $\mathrm{H}^{+}$-ions through the membrane. Therefore we can describe the change of $\Delta \mathrm{pH}$ with time by

$\frac{\mathrm{d}}{\mathrm{d} t} \Delta \mathrm{pH}=k_{13} \cdot\left(f_{0}+f_{5}+f_{6}+f_{11}\right)-k_{14} \cdot \Delta \mathrm{pH}$.

Due to the $\mathrm{pH}$-difference across the membrane ATP is synthesized from ADP. On the other hand 3 ATP molecules are used to bind one $\mathrm{CO}_{2}$-molecule, i.e. for every four electrons transported from $\mathrm{NADPH}_{2}$ to the intermediates of the dark reaction. Therefore we can describe the ATP-formation by

$$
\frac{\mathrm{d}}{\mathrm{d} t}[\mathrm{ATP}]=k_{15} \cdot \Delta \mathrm{pH} \cdot[\mathrm{ADP}]-\frac{3}{4} \cdot f_{12} .
$$

$f_{\mathrm{i}}$ are the electron transport rates and $k_{\mathrm{i}}$ are the transport coefficients according to Fig. 1.

For the stationary state we have $f_{0}=f_{5}=f_{6}=f_{12}=$ $f_{\mathrm{e}}$ and

and

$$
\Delta \mathrm{pH}_{\mathrm{s}}=\frac{k_{13}}{k_{14}} \cdot 4 \cdot f_{\mathrm{e}}
$$

$$
[\mathrm{ATP}]_{\mathrm{s}}=\left[\mathrm{ADP}_{0}\right]-\frac{3}{16} \cdot \frac{k_{14}}{k_{13} \cdot k_{15}} .
$$

The stationary electron flow rate $f_{\mathrm{e}}$ is determined by the chosen poolsize of the intermediates and by the experimentally known reduction-time and reoxidation-time of plastoquinone [13-15]. The poolsize of plastoquinone is set to be $\left[\mathrm{PQ}_{0}\right]=500$, which implies that the sizes of the other pools $(Z, Q, X$, Cyt-f, P700, FD, PSII, and PSI) are 100, i.e. we consider 100 electron transport chains. 
This results in $f_{\mathrm{e}} \sim 9000, i . e$. the absolute value of the stationary electron transport is $\sim 90 \mathrm{e} / \mathrm{sec}$ in one chain.

This is in agreement with reported values [22], if we take into account that one chain has about 500 chlorophyllmolecules (250 in PSII and 250 in PSI). Since four electrons are transported for each $\mathrm{O}_{2}$-molecule released, this implies that we have an oxygen evolution of about $20-25 \mathrm{O}_{2}$-molecules/sec in one chain. This is twice the value reported in ref. [23].

The stationary rate of ATP-formation is calculated to be $60-75$ ATP-molecules/sec per chain.

Of course, these values are sensitive to light intensity, to the stationary $\Delta \mathrm{pH}$-value, and to the values of the coefficients chosen in the model.

From the experimentally known values of $\Delta \mathrm{pH}_{\mathrm{s}}$ [13] then the ratio $k_{13} / k_{14}$ is also determined (e.g. for $\Delta \mathrm{pH}_{\mathrm{s}}=2.2$ is $\left.k_{13} / \mathrm{k}_{14} \sim 6 \times 10^{-5}\right)$. On the other hand we also know the decay time of $\Delta \mathrm{pH}$ in the dark when electron flow stops [13]. The time constant is measured roughly to be $k_{14}=1.0 \mathrm{sec}^{-1}$, i.e. $k_{13}=6 \times 10^{-5}$. The coefficient $k_{15}$ determines the ATP-formation and thus the position of the Ppeak in the fluorescence induction curve. If $k_{15}$ is too small then the P-S-decrease will occur too late in the model compared with the experimental curve. If $k_{15}$ is too high then the P-S-decrease will occur too early or even cause the P-peak to disappear if the electron flow in the model is increased by the reoxidation of the quencher $\mathrm{Q}$ before the pile up process dominates.

From this a value of $k_{15} \sim 1.5 \times 10^{3}$ is evaluated from the model comparing with the experimental fluorescence induction curve.

Using these values the computer model shows that the $\Delta \mathrm{pH}$-value changes relatively slowly - the characteristic time is about $1 \mathrm{sec}$, whereas the ATP-value almost immediately follows the $\Delta \mathrm{pH}$-value.

We have to be aware of the fact that the $\Delta \mathrm{pH}$ value of the model corresponds to the total electrochemical potential difference across the thylakoid membrane, i.e. the real $\mathrm{pH}$-difference and also the potential difference $\Delta \psi$ across the membrane [13, $16]$, which is also designated "proton motive force" (pmf) [8].

\section{Results and Comparison with the Model}

Increasing the time of dark adaptation prior to the measurement results in an increase of the peak $\mathrm{P}$ in the fluorescence induction curve. At short times of dark adaptation (seconds) a fluorescence induction curve without the peak $\mathrm{P}$ is obtained. Fig. 2 shows the P-S-difference in dependence on the time of dark adaptation. Since the peak P is suggested to result from the low energy state and since the ATP level decreases in the dark in some species in the same time scale as the peak $P$ occurs [17] we may suggest that the increase of peak $\mathrm{P}$ with time of dark adaptation is caused by a decrease of the ATPlevel due to a decay of the electrochemical potential leading to a stop of ATP-synthesis. This assumption was checked with the computer model - in order to check the consistency of the model. All the pools were set to have the state of the dark adapted system, since the electron transfer between the pools takes very short time, about $\mathrm{ms}$, compared to the dark adaptation time considered here. Only the initial $\triangle \mathrm{pH}$-value was set to different values. Fig. 3 shows the calculated P-S-difference in dependence on the initial $\Delta \mathrm{pH}$-value of the system.

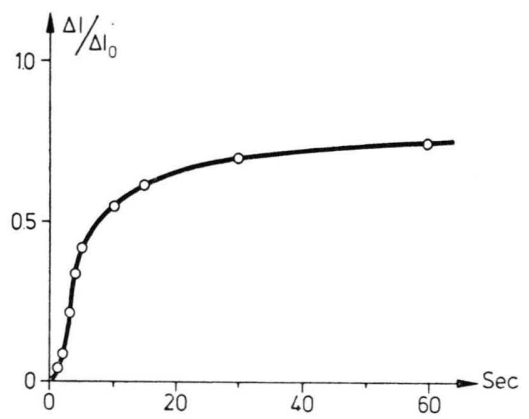

Fig. 2. Difference $\Delta \mathrm{I}$ between the intensity of the fluorescence of the P-peak and the fluorescence of the quasisteady S-level divided by $\Delta \mathrm{I}_{0}$ of a fully dark adapted system, measured with Chlorella vulgaris in dependence on the time of dark adaptation.

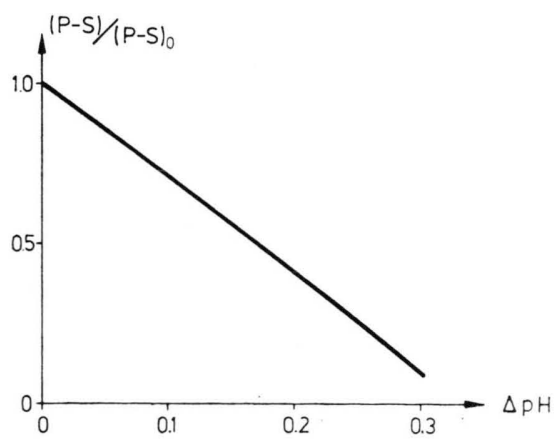

Fig. 3. The calculated P-S-difference in dependence on the initial $\Delta \mathrm{pH}$-value divided by the P-S-difference at $\Delta \mathrm{pH}_{\text {init }}$ $=0$. 


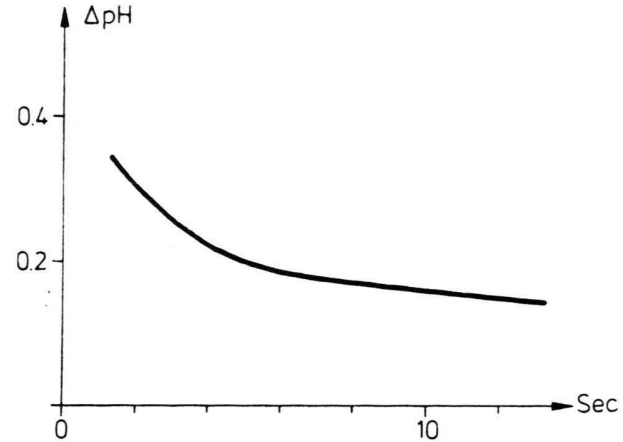

Fig. 4. Decrease of the hypothetical electrochemical potential $\triangle \mathrm{pH}$ during dark adaptation of the photosynthetic system.

Comparison of the Figs 2 and 3 yields the decrease of the hypothetical electrochemical potential $\Delta \mathrm{pH}$ in the dark (Fig. 4).

If we compare this with the measured decay of the real $\mathrm{pH}$-gradient according to Witt [13] with a decay time of $1 \mathrm{sec}$, then we see that the hypothetical electrochemical potential $\Delta \mathrm{pH}$, although the time scale is the same, decreases more quickly in the first few seconds and than decreases more slowly than the experimental $\mathrm{pH}$-gradient.

The $\mathrm{P} \rightarrow \mathrm{S}$ depression seems to be determined mainly by the $\mathrm{pH}$-gradient, but also influenced by some other factors with a slower decay than the $\mathrm{pH}$ gradient, perhaps by some residual ATP.

\section{Discussion}

The P-S-decrease in fluorescence intensity has been related to an increase of electron transport and reoxidation of the quencher due to starting of dark reaction, uptake of $\mathrm{CO}_{2}$, and Calvin-cycle, or due to an increase in PSI activity [18]. Slow fluorescence induction phenomena in the time range up to minutes which are not considered in this paper, have been related to the photophosphorylation process and to structural change in the thylakoid membrane $[2,19]$. The increase of PSI activity during the P-S-decrease or the starting of the Calvin-cycle, probably needs some sources of energy. This may be the membrane potential, or the $\mathrm{pH}$-difference across the membrane of the thylakoids built up by the proton pumping effect of the light driven electron transport or by ATP. Therefore, also a correlation between the P-S-decrease and a high energy state production is suggested as described earlier [2]. As explained above the simple model of the reoxidation of the intermediates of the electron transport chain roughly describes the P-S-decrease if the hypothetical parameter $\Delta \mathrm{pH}$ in the model is considered to describe the high energy state.

Therefore, the uncoupler $\mathrm{NH}_{4} \mathrm{Cl}$ which is believed to collapse the $\mathrm{pH}$-gradient [8] should inhibit the P-S-fluorescence quenching. Fig. 5 shows the influence of $\mathrm{NH}_{4} \mathrm{Cl}$ on the fluorescence induction curve. In fact, the S-line is rised with $\mathrm{NH}_{4} \mathrm{Cl}$ without affecting the P-peak very much [2].

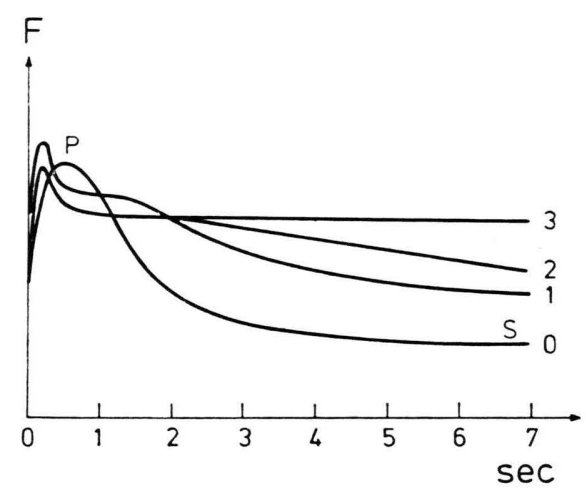

Fig. 5. Influence of $\mathrm{NH}_{4} \mathrm{Cl}$ on the fluorescence induction curve of Chlorella vulgaris. $0 \ldots$ Control; $1 \ldots 20 \mathrm{~mm} / 1$ $\mathrm{NH}_{4} \mathrm{Cl} ; 2 \ldots 40 \mathrm{~mm} / \mathrm{l} \mathrm{NH}_{4} \mathrm{Cl} ; 3 \ldots 80 \mathrm{~mm} / 1 \mathrm{NH}_{4} \mathrm{Cl} . \mathrm{P} \ldots$ P-peak of the control curve; S... Steady state of the control curve.

There are still some difficulties with this picture concerning the effect of other uncouplers of phosphorylation. Also the uncoupler CCCP is believed to colapse the transmembrane $\mathrm{pH}$-difference. This should prevent ATP production, i.e. the P-S-decrease should be inhibited. However, this seems not to be the case. CCCP has no effect on the fast fluorescence induction curve at low concentrations, where it acts as an uncoupler of phosphorylation $\left(\sim 10^{-7}-10^{-6}\right.$ $\mathrm{mol} / \mathrm{l})[4,20]$. That might be due to the fact that there exists a threshold effect in the ATP formation in dependence on the electrochemical gradient. If the electrochemical gradient is not collapsed totally there might be enough energy, e.g. in the transmembrane potential itself which is not collapsed by neutral molecules penetrating the membrane, as for example $\mathrm{NH}_{3}$, to produce ATP leading to a P-Sdecrease.

The threshold effect is explained by Gräber and Witt [16] by a different fractional dependence of a basal proton flux and a phosphorylation proton 
flux. In this computer model the threshold effect is explained by the coupling of the electron transport through the intermediates and the phosphorylation according to Eqns (1) and (2). Although Eqn (2) does not explicitely exhibit any threshold effect the coupling by the second term to the electron transport $f_{12}$ leads to a threshold effect.

The electron transport $f_{12}$ due to reoxidation of $\mathrm{NADPH}_{2}$ is coupled to the pools by the simple assumption

$$
f_{12}=k_{12} \cdot\left[\mathrm{NADPH}_{2}\right] \cdot[\mathrm{ATP}]
$$

and the $\left[\mathrm{NADPH}_{2}\right]$-pool is coupled to the other pools with electron transport by similar equations $[9,10]$.

If the steady state values are calculated for different constant $\Delta \mathrm{pH}$-values, which corresponds to the case that $\Delta \mathrm{pH}$ has a certain value in the S-state depending on the $\mathrm{H}^{+}$-leak due to uncouplers present, i.e. depending on $k_{14}$ in Eqn (1), then we obtain for e.g. $[\mathrm{ATP}]_{\mathrm{s}},\left[\mathrm{NADPH}_{2}\right]$, electron transport rate $f_{\mathrm{e}}$, and the quencher $\left[\mathrm{Q}^{-}\right]_{\mathrm{s}}$ the dependence in Fig. 6.

$[\mathrm{ATP}]_{\mathrm{s}}$ is very small below the threshold value of $\Delta \mathrm{pH} \sim 0.4$. Below that value also the redoxstate of the quencher $\mathrm{Q}$ depends on $\Delta \mathrm{pH}$. With decreasing $\Delta \mathrm{pH}\left[\mathrm{Q}^{-}\right]_{\mathrm{s}}$ is increased which means an inhibition of the P-S-decrease in the fluorescence induction curve. Above the threshold value the $[\mathrm{ATP}]_{\mathrm{s}}$ value increases rapidly and the $\left[\mathrm{NADPH}_{2}\right]_{\mathrm{s}}$ value decreases leading to a value of $\left[\mathrm{Q}^{-}\right]_{\mathrm{s}}$ which is not influenced by the $\triangle \mathrm{pH}$. That means that an uncoupler only can inhibit the P-S-decrease in the fluorescence induction curve if the hypothetical electrochemical parameter $\Delta \mathrm{pH}$ is suppressed below the threshold value of about 0.4 . That may not be the case with some inhibitors especially at low concentrations if they only collaps the real $\mathrm{pH}$-gradient and not the membrane potential $\Delta \psi$. Thus it may be possible that still some ATP is produced by the potential difference $[16,21]$ which may be the

[1] S. Malkin and B. Kok, Biochim. Biophys. Acta 126, 413-432 (1966)

[2] G. Papageorgiou, Bioenergetics of Photosynthesis (Govindjee, ed.), Academic Press, 1975.

[3] R. K, Clayton, Light and Living Matter, McGraw-Hill Book Comp., New York 1971.

[4] R. Bauer, Thesis TH-Aachen, 1972.

[5] D. S. Bendall and R. Hill, Ann. Rev. Plant Physiol. 19, 167-186 (1968).

[6] P. Mitchell, Glynn Research Laboratories, 1966.

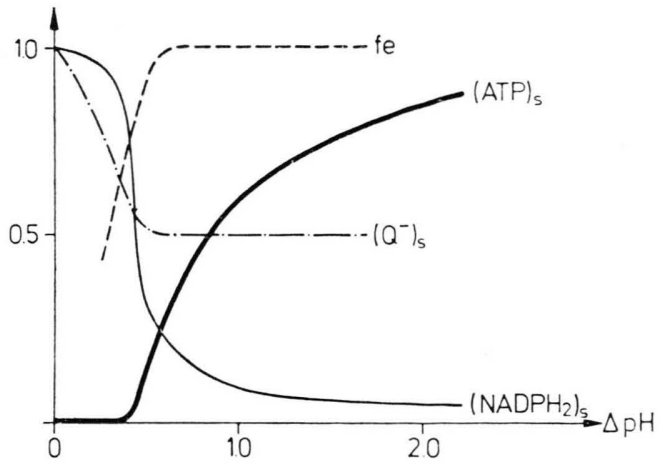

Fig. 6. Calculated steady state values of ATP, $\mathrm{NADPH}_{2}$, and reduced quencher $\mathrm{Q}^{-}$divided by their total poolsize, and calculated steady state value of the electron transport rate divided by the $\Delta \mathrm{pH}$ independent value above $\Delta \mathrm{pH}=0.5$ in dependence of the $\Delta \mathrm{pH}$ value. In the computer model was $\Delta \mathrm{pH}=$ const. set by $k_{13}=0$ and $k_{14}=0$ and by different initial values of $\Delta \mathrm{pH}$. With these values the induction curves of ATP, NADPH $\mathrm{N}_{2}$, and $\mathrm{Q}^{-}$were calculated. The steady state was obtained after a time of calculation corresponding to about $0.3 \mathrm{sec}$.

reason that the P-S-quenching is not totally inhibited with $\mathrm{NH}_{4} \mathrm{Cl}$ (Fig. 5).

We have used the simple linear relation between $\triangle \mathrm{pH}$ and ATP-formation [Eqn (2)]. Using an exponential relation according to Gräber and Witt [16] only leads to a slight change in the curves in Figs 3 and 4. Also the threshold effect in Fig. 6 is insensitiv to the special relationship between $\Delta \mathrm{pH}$ and ATP-formation. The threshold effect is much more a consequence of the coupling between the ATP-formation and the electron transport.

The author wishes to thank U. Zimmermann for the support of this work and for many helpful discussions.

The author also wishes to thank Mrs. S. Alexowsky for typewriting and Chr. Matschke for skillful technical assistance especially in construction of the algae growing facility.

[7] P. Mitchell, Glynn Research Laboratories, 1968.

[8] A. T. Jagendorf, Bioenergetics of Photosynthesis (Govindjee, ed.), Academic Press, 1975.

[9] Chr. Holzapfel and R. Bauer, Z. Naturforsch. 30 c, 489-498 (1975).

[10] Chr. Holzapfel, Jül-1390, KFA Jülich, 1977.

[11] R. Bauer and M. J. G. Wijnands, Z. Naturforsch. 29 c, $725-732$ (1974).

[12] A. Trebst and S. Reimer, Z. Naturforsch. 28 c, $710-$ $716(1973)$ 
[13] H. T. Witt, Quart. Rev. Biophysics 4, 365 (1971).

[14] H. T. Witt, Bioenergetics of Photosynthesis (Govindjee, ed.), Academic Press, 1975.

[15] H. H. Stiehl, Thesis TU Berlin, 1969.

[16] P. Gräber and H. T. Witt, Biochim. Biophys. Acta 423, 141-163 (1976).

[17] U. Heber and K. A. Santorius, Z. Naturforsch. 25 b, $718-728$ (1970).

[18] R. Bauer and U. F. Franck, Proc. IIIrd Int. Congr. Photosynthesis, Rehovot, Israel (M. Avron, ed.), Elsevier Scient. Publ. Comp., Amsterdam 1974.
[19] G. Papageorgiou and Govindjee, Biophys. J. 8, 13161328 (1968).

[20] M. J. G. Wijnands, Thesis TH Aachen, 1974.

[21] W. Junge, B. Rumberg, and H. Schröder, Eur. J. Biochem. 41, 575-581 (1970).

[22] U. Siggel, Diss. TU Berlin, 1974.

[23] E. Rabinowitch and Govindjee, Photosynthesis, John Wiley and Sons Inc., New York 1969.

[24] A. Kuhl and M. Lorenzen, Methods in Cell Physiology (D. M. Prescott, ed.), Vol. I, p. 159-180, Academic Press, New York 1964. 